\title{
Extended domain for fifth convergence order schemes
}

\author{
IOANNIS K. Argyros ${ }^{1}$ \\ SAnthosh George ${ }^{2}$ \\ 1 Department of Mathematical Sciences, \\ Cameron University, Lawton, OK 73505, \\ USA. \\ iargyros@cameron.edu \\ 2 Department of Mathematical and \\ Computational Sciences, NIT Karnataka, \\ India-575 025. \\ sgeorge@nitk.edu.in
}

\begin{abstract}
We provide a local as well as a semi-local analysis of a fifth convergence order scheme involving operators valued on $\mathrm{Ba}$ nach space for solving nonlinear equations. The convergence domain is extended resulting a finer convergence analysis for both types. This is achieved by locating a smaller domain included in the older domain leading this way to tighter Lipschitz type functions. These extensions are obtained without additional hypotheses. Numerical examples are used to test the convergence criteria and also to show the superiority for our results over earlier ones. Our idea can be utilized to extend other schemes using inverses in a similar way.
\end{abstract}

\section{RESUMEN}

Entregamos un análisis local y uno semi-local de un esquema de quinto orden de convergencia que involucra operadores con valores en un espacio de Banach para resolver ecuaciones nolineales. El dominio de convergencia es extendido resultando en un análisis de convergencia más fino para ambos tipos. Esto se logra ubicando un dominio más pequeño incluido en el dominio antiguo, entregando funciones de tipo Lipschitz más ajustadas. Estas extensiones se obtienen sin hipótesis adicionales. Se usan ejemplos numéricos para verificar los criterios de convergencia y también para mostrar que nuestros resultados son superiores a otros anteriores. Nuestra idea se puede utilizar para extender otros esquemas usando inversos de manera similar.

Keywords and Phrases: Fifth order convergence scheme, w-continuity, convergence analysis, Fréchet derivative, Banach space.

2020 AMS Mathematics Subject Classification: 65H10, 47H17, 49M15, 65D10, 65G99. 


\section{Introduction}

In this article, $B_{1}, B_{2}$ are standing for Banach spaces, $D \subset B_{1}$ is denoting a convex and open set, and $F: D \longrightarrow B_{2}$ is considered differentiable according to the Fréchet notion. One of the most important tasks is the location of a solution $x_{*}$ of nonlinear equation

$$
F(x)=0 .
$$

Solving equation $F(x)=0$ is useful because using modeling (Mathematical) problems from many areas can be formulated as (1.1). The explosion of technology requires the development of higher convergence schemes. Starting from the quadratically convergent Newton's method higher order schemes develop all the time $[1,2,3,4,5,6,7,8,9,10,11,12,13,14,15,16,17,18,19]$.

Recently, Singh et al. [13] provided a semi-local convergence for efficient fifth order scheme under Lipschitz continuity on $F^{\prime \prime}$ defined as follows

$$
\begin{aligned}
y_{n} & =x_{n}-F^{\prime}\left(x_{n}\right)^{-1} F\left(x_{n}\right) \\
z_{n} & =y_{n}-F^{\prime}\left(x_{n}\right)^{-1} F\left(y_{n}\right) \\
x_{n+1} & =z_{n}-F^{\prime}\left(y_{n}\right)^{-1} F\left(z_{n}\right) .
\end{aligned}
$$

Later in [14] the applicability of scheme (1.2) was extended using w- continuity conditions. In general, the convergence domain is small. That is why we develop a technique where a tighter domain than before is obtained containing the iterates. This way the new w-functions are tighter leading to a finer semi-local convergence analysis. It is worth noticing that these extensions do not involve new hypotheses because the new w-functions are specializations of the old one. Hence, we extend the applicability of the method. It turns out that the local convergence analysis can be extended too.

For example: Let $B_{1}=B_{2}=\mathbb{R}, \Omega=\left[-\frac{1}{2}, \frac{3}{2}\right]$. Define $G$ on $\Omega$ by

$$
G(x)=\left\{\begin{array}{cc}
x^{3} \log x^{2}+x^{5}-x^{4}, & x \neq 0 \\
0, & x=0 .
\end{array}\right.
$$

Then, we get $x_{*}=1$, and

$$
\begin{gathered}
G^{\prime}(x)=3 x^{2} \log x^{2}+5 x^{4}-4 x^{3}+2 x^{2}, \\
G^{\prime \prime}(x)=6 x \log x^{2}+20 x^{3}-12 x^{2}+10 x, \\
G^{\prime \prime \prime}(x)=6 \log x^{2}+60 x^{2}-24 x+22 .
\end{gathered}
$$

Obviously $G^{\prime \prime \prime}(x)$ is not bounded on $\Omega$. So, the convergence of scheme (1.2) is not guaranteed by the analysis in $[13,14]$. In this study we use only assumptions on the first derivative to prove our results. Relevant studies can be found in [6, 19].

The structure of the rest of the article involves local and semi-local convergence analysis in Section 2 and Section 3, respectively. The numerical experiments appear in Section 4. 


\section{Local convergence}

It is easier for the local convergence of method (1.2), if we develop some real functions. We start with a function $\omega_{0}$ defined on the interval $I=[0, \infty)$ with values in $I$ satisfying $\omega_{0}(0)=0$. Assume equation

$$
\omega_{0}(t)=1
$$

has a least positive solution called $\rho_{0}$. Assume the existence of function $\omega$, continuous increasing defined on $I_{0}=\left[0, \rho_{0}\right)$ with values in $I$ satisfying $\omega(0)=0$. Define functions $\lambda_{1}$ and $\mu_{1}$ on $I_{0}$ as follows

$$
\lambda_{1}(t)=\frac{\int_{0}^{1} \omega((1-\theta) t) d \theta}{1-\omega_{0}(t)}
$$

and

$$
\mu_{1}(t)=\lambda_{1}(t)-1 .
$$

These definitions lead to $\mu_{1}(0)=-1$ and $\mu_{1}(t) \longrightarrow \infty$ as $t \longrightarrow \rho_{0}^{-}$. Then, the theorem on intermediate value assure the existence of solutions for the equation $\mu_{1}(t)=0$ in $\left(0, \rho_{0}\right)$. Set $R_{1}$ to be the least such solution. Assume equation

$$
\omega_{0}\left(\lambda_{1}(t) t\right)=1
$$

has a least positive solution called $\rho_{1}$. Set $I_{1}=\left[0, \rho_{2}\right), \rho_{2}=\min \left\{\rho_{0}, \rho_{1}\right\}$. Define functions $\lambda_{2}$ and $\mu_{2}$ on $I_{1}$ as follows

$$
\lambda_{2}(t)=\frac{\int_{0}^{1} \omega\left((1-\theta) \lambda_{1}(t) t\right) d \theta \lambda_{1}(t)}{1-\omega_{0}\left(\lambda_{1}(t) t\right)}
$$

and

$$
\mu_{2}(t)=\lambda_{2}(t)-1 .
$$

This time we also have $\lambda_{2}(0)=-1$ and $\lambda_{2}(t) \longrightarrow \infty$ as $t \longrightarrow \rho_{2}^{-}$. Call $R_{2}$ the smallest solution of equation $\lambda_{2}(t)=0$ in $\left(0, \rho_{2}\right)$. Assume equation

$$
\omega_{0}\left(\lambda_{2}(t) t\right)=1
$$

has a least positive solution called $\rho_{3}$. Set $I_{2}=\left[0, \rho_{4}\right), \rho_{4}=\min \left\{\rho_{2}, \rho_{3}\right\}$. Consider functions $\lambda_{3}$ and $\mu_{3}$ on $I_{2}$ as follows

$$
\lambda_{3}(t)=\left[\frac{\int_{0}^{1} \omega\left((1-\theta) \lambda_{2}(t) t\right) d \theta}{1-\omega_{0}\left(\lambda_{2}(t) t\right)}+\frac{\left(\omega_{0}\left(\lambda_{2}(t) t\right)+\omega_{0}\left(\lambda_{1}(t) t\right) \int_{0}^{1} v\left(\theta \lambda_{2}(t) t\right) d \theta\right.}{\left(1-\omega_{0}\left(\lambda_{2}(t) t\right)\right)\left(1-\omega_{0}\left(\lambda_{1}(t) t\right)\right)}\right] \lambda_{2}(t)
$$

and

$$
\mu_{3}(t)=\lambda_{3}(t)-1
$$


where $v: I_{2} \longrightarrow I$ is an increasing and continuous function. By these functions, we obtain $\mu_{3}(0)=-1$ and $\mu_{3}(t) \longrightarrow \infty$ as $t \longrightarrow \rho_{4}^{-}$. Let $R_{3}$ stand for the smallest solution of equation $\mu_{3}(t)=0$ in $\left(0, \rho_{4}\right)$. A radius of convergence can be given as follows

$$
R=\min \left\{R_{i}\right\}, i=1,2,3
$$

Then, for all $t \in[0, R)$.

$$
\begin{aligned}
& 0 \leq \omega_{0}(t)<1 \\
& 0 \leq \omega_{0}\left(\lambda_{1}(t) t\right)<1 \\
& 0 \leq \omega_{0}\left(\lambda_{1}(t) t\right)<1 \\
& 0 \leq \omega_{0}\left(\lambda_{2}(t) t\right)<1
\end{aligned}
$$

and

$$
0 \leq \lambda_{i}(t)<1
$$

Denote by $U\left(x_{*}, \gamma\right)$ a ball of center $x_{*}$ and with a radius $\gamma>0$. Then, $\bar{U}\left(x_{*}, \gamma\right)$ stands for the closure of $U\left(x_{*}, \gamma\right)$.

We base the local convergence on this notation and the conditions $(\mathrm{C})$.

(c1) $F: D \longrightarrow B_{2}$ is differentiable according to Fréchet, and $x_{*} \in D$ with $F\left(x_{*}\right)=0$ is a simple solution.

(c2) There exists an increasing and continuous real function $\omega_{0}$ on $I$ satisfying $\omega_{0}(0)=0$ and such that for all $x \in D$

$$
\left\|F^{\prime}\left(x_{*}\right)^{-1}\left(F^{\prime}(x)-F^{\prime}\left(x_{*}\right)\right)\right\| \leq \omega_{0}\left(\left\|x-x_{*}\right\|\right) .
$$

Set $U_{0}=D \cap U\left(x_{*}, \rho_{0}\right)$.

(c3) There exists a function $\omega$ on $I_{0}$ continuous and increasing satisfying $\omega(0)=0$ such that for all $x, y \in U_{0}$

$$
\left\|F^{\prime}\left(x_{*}\right)^{-1}\left(F^{\prime}(y)-F^{\prime}(x)\right)\right\| \leq \omega(\|y-x\|) .
$$

Set $U_{1}=D \cap U\left(x_{*}, \rho_{4}\right)$.

(c4) There exists a function $v$ on $I_{2}$ continuous and increasing, such that for all $x \in U_{1}$

$$
\left\|F^{\prime}\left(x_{*}\right)^{-1} F^{\prime}(x)\right\| \leq v\left(\left\|x-x_{*}\right\|\right) .
$$

$(\mathrm{c} 5) \bar{U}\left(x_{*}, R\right) \subseteq D$.

(c6) There exists $R_{1} \geq R$ such that

$$
\int_{0}^{1} \omega_{0}\left(\theta R_{1}\right) d \theta<1
$$

Set $U_{2}=D \cap \bar{U}\left(x_{*}, R_{1}\right)$. 
Theorem 2.1. Assume hypotheses (C) hold and starting point $x_{0} \in U\left(x^{*}, R\right)-\left\{x^{*}\right\}$. Then the following assertions are valid, sequence $\left\{x_{n}\right\}$ belongs in $U\left(x_{*}, R\right)-\left\{x_{*}\right\}$ and converges to $x_{*} \in$ $U\left(x_{*}, R\right)$ so that this limit point uniquely solves equation $F(x)=0$ in the set $U_{2}$.

Proof. Let $z \in U\left(x^{*}, R\right)-\left\{x_{*}\right\}$ and utilize (c2), (2.4) and (2.5) to obtain

$$
\left\|F^{\prime}\left(x_{*}\right)^{-1}\left(F^{\prime}(z)-F^{\prime}\left(x_{*}\right)\right)\right\| \leq \omega_{0}\left(\left\|z-x_{*}\right\|\right) \leq \omega_{0}(R)<1,
$$

which together with a result by Banach [12] for linear operators whose inverse exists imply

$$
\left\|F^{\prime}(z)^{-1} F^{\prime}\left(x_{*}\right)\right\| \leq \frac{1}{1-\omega_{0}\left(\left\|z-x^{*}\right\|\right)} .
$$

In particular, by scheme (1.2) $y_{0}, z_{0}$ are well defined since if we set $z=x_{0} \in U\left(x_{*}, R\right)-\left\{x_{*}\right\}$, and $F^{\prime}\left(x_{0}\right)$ is invertible. Then, by (2.4), (2.8) (for $k=1$ ), (c1), (c3) and (2.10) (for $z=x_{0}$ ), we have

$$
\begin{aligned}
\left\|y_{0}-x_{*}\right\| & =\left\|x_{0}-x_{*}-F^{\prime}\left(x_{0}\right)^{-1} F\left(x_{0}\right)\right\| \\
& \leq\left\|F^{\prime}\left(x_{0}\right)^{-1} F^{\prime}\left(x_{*}\right)\right\|\left[\int_{0}^{1}\left\|F^{\prime}\left(x_{*}\right)^{-1}\left[F^{\prime}\left(x_{0}+\theta\left(x_{0}-x_{*}\right)\right)-F^{\prime}\left(x_{0}\right)\right]\left(x_{0}-x_{*}\right) d \theta\right\|\right] \\
& \leq \frac{\int_{0}^{1} \omega\left((1-\theta)\left\|x_{0}-x_{*}\right\|\right) d \theta}{1-\omega_{0}\left(\left\|x_{0}-x_{*}\right\|\right)}\left\|x_{0}-x_{*}\right\| \\
& \leq \lambda_{1}\left(\left\|x_{0}-x_{*}\right\|\right)\left\|x_{0}-x_{*}\right\| \leq\left\|x_{0}-x_{*}\right\|<R .
\end{aligned}
$$

Hence, $y_{0} \in U\left(x_{*}, R\right)$. Using the second substep of method (1.2) and replacing $x_{0}, y_{0}$, by $y_{0}, z_{0}$, respectively as in (2.10) and (2.11), we get

$$
\begin{aligned}
\left\|z_{0}-x_{*}\right\| & \leq \frac{\int_{0}^{1} \omega\left((1-\theta)\left\|y_{0}-x_{*}\right\|\right) d \theta}{1-\omega_{0}\left(\left\|y_{0}-x_{*}\right\|\right)}\left\|y_{0}-x_{*}\right\| \\
& \leq \frac{\int_{0}^{1} \omega\left((1-\theta) \lambda_{1}\left(\left\|x_{0}-x_{*}\right\|\right)\left\|x_{0}-x_{*}\right\|\right) d \theta \lambda_{1}\left(\left\|x_{0}-x_{*}\right\|\right)\left\|x_{0}-x_{*}\right\|}{1-\omega_{0}\left(\lambda_{1}\left(\left\|x_{0}-x_{*}\right\|\right)\left\|x_{0}-x_{*}\right\|\right.} \\
& \leq \lambda_{2}\left(\left\|x_{0}-x_{*}\right\|\right)\left\|x_{0}-x_{*}\right\| \leq\left\|x_{0}-x_{*}\right\| .
\end{aligned}
$$

That is $z_{0} \in U\left(x_{*}, R\right)$ and also $x_{1}$ exists (for $y_{0}=z$, in (2.10)). Notice that (c1), (c4), (2.12) and

$$
F\left(z_{0}\right)=F\left(z_{0}\right)-F\left(x_{*}\right)=\int_{0}^{1} F^{\prime}\left(x_{*}+\theta\left(z_{0}-x_{*}\right)\right) d \theta\left(z_{0}-x_{*}\right)
$$

we obtain that

$$
\begin{aligned}
& \left\|F^{\prime}\left(x_{*}\right)^{-1} F^{\prime}\left(z_{0}\right)\right\| \\
\leq & \int_{0}^{1} v\left(\theta\left\|z_{0}-x_{*}\right\|\right) d \theta\left\|z_{0}-x_{*}\right\| \\
\leq & \int_{0}^{1} v\left(\theta \lambda_{2}\left(\left\|x_{0}-x_{*}\right\|\right)\left\|x_{0}-x_{*}\right\| d \theta \lambda_{2}\left(\left\|x_{0}-x_{*}\right\|\right)\left\|x_{0}-x_{*}\right\| .\right.
\end{aligned}
$$

Moreover, by the last substep of method (1.2), (2.4), (2.5), (2.8) (for $k=3$ ), (2.10), (2.13) (for 
$\left.z=x_{0}, y_{0}\right),(2.11)$ and $(2.12)$, we have in turn that

$$
\begin{aligned}
\left\|x_{1}-x_{*}\right\| \leq & \left\|z_{0}-x_{*}-F^{\prime}\left(z_{0}\right)^{-1} F\left(z_{0}\right)\right\| \\
& +\left\|F^{\prime}\left(z_{0}\right)^{-1}\left[\left(F^{\prime}\left(y_{0}\right)-F^{\prime}\left(x_{*}\right)\right)+\left(F^{\prime}\left(x_{*}\right)-F^{\prime}\left(z_{0}\right)\right)\right] F^{\prime}\left(y_{0}\right)^{-1} F\left(z_{0}\right)\right\| \\
\leq & {\left[\frac{\int_{0}^{1} \omega\left((1-\theta)\left\|z_{0}-x_{*}\right\|\right) d \theta}{1-\omega_{0}\left(\left\|z_{0}-x_{*}\right\|\right)}\right.} \\
& \left.+\frac{\left(\omega_{0}\left(\left\|z_{0}-x_{*}\right\|\right)+\omega_{0}\left(\left\|y_{0}-x_{*}\right\|\right)\right) \int_{0}^{1} v\left(\theta\left\|z_{0}-x_{*}\right\|\right) d \theta}{\left(1-\omega_{0}\left(\left\|z_{0}-x_{*}\right\|\right)\right)\left(1-\omega_{0}\left(\left\|y_{0}-x_{*}\right\|\right)\right)}\right]\left\|z_{0}-x_{*}\right\| \\
\leq & \lambda_{3}\left(\left\|x_{0}-x_{*}\right\|\right)\left\|x_{0}-x_{*}\right\| \leq\left\|x_{0}-x_{*}\right\|,
\end{aligned}
$$

so $x_{1} \in U\left(x_{*}, R\right)$. Replacing $x_{0}, y_{0}, z_{0}, x_{1}$ by $x_{k}, y_{k}, z_{k}, x_{k+1}$, in the previous computations we obtain

$$
\begin{gathered}
\left\|y_{k}-x_{*}\right\| \leq \lambda_{1}\left(\left\|x_{k}-x_{*}\right\|\right)\left\|x_{k}-x_{*}\right\| \leq\left\|x_{k}-x_{*}\right\|<R, \\
\left\|z_{k}-x_{*}\right\| \leq \lambda_{2}\left(\left\|x_{k}-x_{*}\right\|\right)\left\|x_{k}-x_{*}\right\| \leq\left\|x_{k}-x_{*}\right\|
\end{gathered}
$$

and

$$
\left\|x_{k+1}-x^{*}\right\| \leq \lambda_{3}\left(\left\|x_{k}-x^{*}\right\|\right)\left\|x_{k}-x_{*}\right\| \leq\left\|x_{k}-x_{*}\right\|
$$

so $y_{k}, z_{k}, x_{k+1}$ stay in $U\left(x_{*}, R\right)$ and $\lim _{k \longrightarrow \infty} x_{k}=x_{*}$. Furthermore, let $x_{*}^{1} \in U_{2}$ with $F\left(x_{*}^{1}\right)=0$. In view of (c2) and (c6) we obtain

$$
\begin{aligned}
\left\|F^{\prime}\left(x_{*}\right)^{-1}\left(\int_{0}^{1} F^{\prime}\left(x_{*}+\theta\left(x_{*}^{1}-x_{*}\right)\right) d \theta-F^{\prime}\left(x_{*}\right)\right)\right\| & \leq \int_{0}^{1} \omega_{0}\left(\theta\left\|x_{*}^{1}-x_{*}\right\|\right) d \theta \\
& \leq \int_{0}^{1} \omega_{0}\left(\theta R_{1}\right) d \theta<1
\end{aligned}
$$

so $x_{*}^{1}=x_{*}$, since $T=\int_{0}^{1} F^{\prime}\left(x_{*}+\theta\left(x_{*}^{1}-x_{*}\right)\right) d \theta$ is invertible and

$$
0=F\left(x_{*}^{1}\right)-F\left(x_{*}\right)=T\left(x_{*}^{1}-x_{*}\right) .
$$

Remark 2.2. 1. In view of (2.10) and the estimate

$$
\begin{aligned}
\left\|F^{\prime}\left(x^{*}\right)^{-1} F^{\prime}(x)\right\| & =\left\|F^{\prime}\left(x^{*}\right)^{-1}\left(F^{\prime}(x)-F^{\prime}\left(x^{*}\right)\right)+I\right\| \\
& \leq 1+\left\|F^{\prime}\left(x^{*}\right)^{-1}\left(F^{\prime}(x)-F^{\prime}\left(x^{*}\right)\right)\right\| \leq 1+L_{0}\left\|x-x^{*}\right\|
\end{aligned}
$$

condition (2.13) can be dropped and $M$ can be replaced by

$$
M(t)=1+L_{0} t
$$

or

$$
M(t)=M=2
$$

since $t \in\left[0, \frac{1}{L_{0}}\right)$. 
2. The results obtained here can be used for operators $F$ satisfying autonomous differential equations [2] of the form

$$
F^{\prime}(x)=P(F(x))
$$

where $P$ is a continuous operator. Then, since $F^{\prime}\left(x^{*}\right)=P\left(F\left(x^{*}\right)\right)=P(0)$, we can apply the results without actually knowing $x^{*}$. For example, let $F(x)=e^{x}-1$. Then, we can choose: $P(x)=x+1$.

3. Let $\omega_{0}(t)=L_{0} t$, and $\omega(t)=L t$. In [2, 3] we showed that $r_{A}=\frac{2}{2 L_{0}+L}$ is the convergence radius of Newton's method:

$$
x_{n+1}=x_{n}-F^{\prime}\left(x_{n}\right)^{-1} F\left(x_{n}\right) \text { for each } n=0,1,2, \cdots
$$

under the conditions (2.11) and (2.12). It follows from the definition of $R$ in (2.4) that the convergence radius $R$ of the method (1.2) cannot be larger than the convergence radius $r_{A}$ of the second order Newton's method (2.19). As already noted in [2, 3] $r_{A}$ is at least as large as the convergence radius given by Rheinboldt [12]

$$
r_{R}=\frac{2}{3 L},
$$

where $L_{1}$ is the Lipschitz constant on $D$. The same value for $r_{R}$ was given by Traub [15]. In particular, for $L_{0}<L_{1}$ we have that

$$
r_{R}<r_{A}
$$

and

$$
\frac{r_{R}}{r_{A}} \rightarrow \frac{1}{3} \text { as } \frac{L_{0}}{L_{1}} \rightarrow 0
$$

That is the radius of convergence $r_{A}$ is at most three times larger than Rheinboldt's.

4. It is worth noticing that method (1.2) is not changing when we use the conditions of Theorem 2.1 instead of the stronger conditions used in [13, 14]. Moreover, we can compute the computational order of convergence (COC) defined by

$$
\xi=\ln \left(\frac{\left\|x_{n+1}-x^{*}\right\|}{\left\|x_{n}-x^{*}\right\|}\right) / \ln \left(\frac{\left\|x_{n}-x^{*}\right\|}{\left\|x_{n-1}-x^{*}\right\|}\right)
$$

or the approximate computational order of convergence

$$
\xi_{1}=\ln \left(\frac{\left\|x_{n+1}-x_{n}\right\|}{\left\|x_{n}-x_{n-1}\right\|}\right) / \ln \left(\frac{\left\|x_{n}-x_{n-1}\right\|}{\left\|x_{n-1}-x_{n-2}\right\|}\right) .
$$

This way we obtain in practice the order of convergence in a way that avoids the bounds involving estimates using estimates higher than the first Fréchet derivative of operator $F$. Note also that the computation of $\xi_{1}$ does not require the usage of the solution $x^{*}$. 


\section{Semi-local convergence analysis}

Let $\Gamma_{0}=F^{\prime}\left(x_{0}\right)^{-1} \in \mathcal{L}\left(B_{2}, B_{1}\right)$ exists at $x_{0} \in D$, where $\mathcal{L}\left(B_{2}, B_{1}\right)$ denotes the set of bounded linear operators from $B_{2}, B_{1}$ and the following conditions hold.

(1) $\left\|\Gamma_{0}\right\| \leq \beta_{0}$.

(2) $\left\|\Gamma_{0} F\left(x_{0}\right)\right\| \leq \eta_{0}$

(3)' $\left\|F^{\prime}(x)-F^{\prime}\left(x_{0}\right)\right\| \leq M_{0}\left\|x-x_{0}\right\|$ for all $x \in D$. Set $D_{0}=D \cap U\left(x_{0}, \frac{1}{\beta_{0} M_{0}}\right)$.

(3) $\left\|F^{\prime \prime}(x)\right\| \leq M$ for all $x \in D_{0}$.

(4) $\left\|F^{\prime \prime}(x)-F^{\prime \prime}(y)\right\| \leq \omega(\|x-y\|)$ for all $x, y \in D_{0}$ for a continuous nondecreasing function $\omega, \omega(0) \geq 0$ such that $\omega(t x) \leq t^{p} \omega(x)$ for $t \in[0,1], x \in(0, \infty)$ and $p \in[0,1]$.

Then, as in $[13,14]$, let $r_{0}=M \beta_{0} \eta_{0}, s_{0}=\beta_{0} \eta_{0} \omega\left(\eta_{0}\right)$ and define sequences $\left\{r_{k}\right\},\left\{s_{k}\right\}$ and $\left\{\eta_{k}\right\}$ for $k=0,1,2, \ldots$, by

$$
\begin{aligned}
r_{k+1} & =r_{k} \varphi\left(r_{k}\right)^{2} \psi\left(r_{k}, s_{k}\right), \\
s_{k+1} & =s_{k} \varphi\left(r_{k}\right)^{2+p} \psi\left(r_{k}, s_{k}\right)^{1+p} \\
\eta_{k+1} & =\eta_{k} \varphi\left(r_{k}\right) \psi\left(r_{k}, s_{k}\right),
\end{aligned}
$$

where

$$
\begin{aligned}
\varphi(t) & =\frac{1}{1-t g(t)} \\
g(t) & =\left(1+\frac{t}{2}+\frac{t^{2}}{2(1-t)}\left(1+\frac{t}{4}\right)\right)
\end{aligned}
$$

and

$$
\begin{aligned}
\psi(t, s)= & \frac{t^{2}}{2(1-t)}\left(1+\frac{t}{4}\right)\left[\frac{s}{1+p}\left(\frac{t^{1+p}}{2^{1+p}}+\frac{1}{2+p}\left(\frac{t^{2}}{2(1-t)}\left(1+\frac{t}{4}\right)\right)^{1+p}\right)\right. \\
& \left.+\frac{t}{2}\left(t+\frac{t^{2}}{2(1-t)}\left(1+\frac{t}{4}\right)\right)\right] .
\end{aligned}
$$

Remark 3.1. In [14] the following conditions were used instead of (3), (4), respectively

(3)' $\left\|F^{\prime \prime}(x)\right\| \leq M_{1}$ for all $x \in D$

(4)' $\left\|F^{\prime \prime}(x)-F^{\prime \prime}(y)\right\| \leq \omega_{1}(\|x-y\|)$ for all $x, y \in D$ and $\omega_{1}$ as $\omega$.

But, we have

$$
D_{0} \subseteq D
$$

so

$$
M_{0} \leq M_{1}
$$




$$
M \leq M_{1}
$$

and

$$
\omega(\theta) \leq \omega_{1}(\theta)
$$

Examples where the preceding items are strict can be found in [1, 2, 3, 4, 5, 6]. Notice that (3)' is used to determine $D_{0}$ leading to $M=M\left(D_{0}, x\right)$. Hence, the results in [13, 14] can be rewritten with $M$ replacing $M_{1}$. So, if $M<M_{1}$ the new semi-local convergence analysis is finer. This is also done under the same computational effort because in practice finding $\omega_{1}, M_{1}$ requires finding $\omega, M_{0}, M$ as special cases. This technique can be used to extend the applicability of other schemes involving inverses in an analogous fashion. Hence, the proof of the following semi-local convergence result for scheme (1.2) is omitted.

Theorem 3.2. Let $r_{0}=M \beta_{0} \eta_{0}<\nu, s_{0}=\beta_{0} \eta_{0} \omega\left(\eta_{0}\right)$ and assumptions (1)-(4) hold. Then, for $\bar{U}\left(x_{0}, R \eta_{0}\right) \subseteq D$, where $R=\frac{g\left(r_{0}\right)}{1-\delta \gamma}$, the sequence $\left\{x_{k}\right\}$ generated by (1.2) converges to the solution $x_{*}$ of $F(x)=0$. Moreover, $y_{k}, z_{k}, x_{k+1}, x_{*} \in \bar{U}\left(x_{0}, R \eta_{0}\right)$ and $x_{*}$ is the unique solution in $U\left(x_{0}, \frac{2}{M_{0} \beta_{0}}-R \eta_{0}\right) \cap D$. Furthermore, we have

$$
\left\|x_{k}-x_{*}\right\| \leq g\left(r_{0}\right) \delta^{k} \frac{\gamma^{\frac{(4+q)^{k}-1}{3+q}}}{1-\delta \gamma^{(4+q)^{k}}} \eta_{0} .
$$

\section{$4 \quad$ Numerical Examples}

Example 4.1. Let us consider a system of differential equations governing the motion of an object and given by

$$
F_{1}^{\prime}(x)=e^{x}, F_{2}^{\prime}(y)=(e-1) y+1, F_{3}^{\prime}(z)=1
$$

with initial conditions $F_{1}(0)=F_{2}(0)=F_{3}(0)=0$. Let $F=\left(F_{1}, F_{2}, F_{3}\right)$. Let $\mathcal{B}_{1}=\mathcal{B}_{2}=\mathbb{R}^{3}, D=$ $\bar{U}(0,1), p=(0,0,0)^{T}$. Define function $F$ on $D$ for $w=(x, y, z)^{T}$ by

$$
F(w)=\left(e^{x}-1, \frac{e-1}{2} y^{2}+y, z\right)^{T}
$$

The Fréchet-derivative is defined by

$$
F^{\prime}(v)=\left[\begin{array}{ccc}
e^{x} & 0 & 0 \\
0 & (e-1) y+1 & 0 \\
0 & 0 & 1
\end{array}\right]
$$

Notice that using the $(\mathcal{A})$ conditions, we get for $\alpha=1, w_{0}(t)=(e-1) t, w(t)=e^{\frac{1}{e-1}} t, v(t)=e^{\frac{1}{e^{-1}}}$. The radii are

$$
\begin{gathered}
R_{1}=0.38269191223238574472986783803208, R_{2}=0.33841523581069998805048726353562, \\
R_{3}=0.32249343047238987480795913143083 \text { and } R=R_{3}
\end{gathered}
$$


Example 4.2. Let $\mathcal{B}_{1}=\mathcal{B}_{2}=C[0,1]$, the space of continuous functions defined on $[0,1]$ be equipped with the max norm. Let $D=\bar{U}(0,1)$. Define function $F$ on $D$ by

$$
F(\varphi)(x)=\varphi(x)-5 \int_{0}^{1} x \theta \varphi(\theta)^{3} d \theta .
$$

We have that

$$
F^{\prime}(\varphi(\xi))(x)=\xi(x)-15 \int_{0}^{1} x \theta \varphi(\theta)^{2} \xi(\theta) d \theta, \text { for each } \xi \in D .
$$

Then, we get that $x^{*}=0$, so $w_{0}(t)=7.5 t, w(t)=15 t$ and $v(t)=2$. Then the radii are

$$
R_{1}=0.066666666666666666666666666666667, R_{2}=0.059338915721683857529278327547217,
$$

$$
R_{3}=0.047722035514509826559237382070933 \text { and } R=R_{3} .
$$

Example 4.3. Returning back to the motivational example at the introduction of this study, we have $w_{0}(t)=w(t)=96.6629073 t$ and $v_{1}(t)=2$. The parameters for method (1.2) are

$$
\begin{gathered}
R_{1}=0.0068968199414654552878434223828208, R_{2}=0.0061008926455964288676492301988219, \\
R_{3}=0.004463243021326804456372361329386 \text { and } R=R_{3} .
\end{gathered}
$$

\section{Conclusion}

In general, the convergence domain of iterative schemes is small limiting their applications. Hence, any attempt to increase it is very important. This is achieved here by finding smaller $\omega$ - functions than before which are also specialization of the previous ones. Hence, the extensions are obtained under the same computational cost. Our idea can be used to extend the usage of other schemes in a similar way. Numerical experiments further demonstrate the superiority of our findings. 


\section{References}

[1] I. K. Argyros, "A new convergence theorem for the Jarratt method in Banach space", Comput. Math. Appl., vol. 36, pp. 13-18, 1998.

[2] I. K. Argyros, Convergence and Application of Newton-Type Iterations, Springer, New York, 2008.

[3] I. K. Argyros, D. Chen, and Q. Qian, "The Jarratt method in Banach space setting", J. Comput. Appl. Math, vol. 51, pp. 103-106, 1994.

[4] I. K. Argyros, and A. A. Magreñañ, Iterative Methods and their dynamics with applications: A Contemporary Study, CRC Press, 2017.

[5] I. K. Argyros, and S. George, Mathematical modeling for the solution of equations and systems of equations with applications, Volume-IV, Nova Publishes, New York, 2020.

[6] M. Chen, Y. Khan, Q. Wu, and A. Yildirim, "Newton-Kantorovich Convergence Theorem of a Modified Newton's Method Under the Gamma-Condition in a Banach Space", Journal of Optimization Theory and Applications, vol. 157, no. 3, pp. 651-662.

[7] J. L. Hueso, and E. Martínez, "Semi-local convergence of a family of iterative methods in Banach spaces", Numer. Algorithms, vol. 67, pp. 365-384, 2014.

[8] A. Kumar, D. K. Gupta, E. Martínez, and S. Singh, "Semi-local convergence of a Steffensen type method under weak Lipschitz conditions in Banach spaces", J. Comput. Appl. Math., vol. 330, pp. $732-741,2018$.

[9] A. A. Magreńãn, "Different anomalies in a Jarratt family of iterative root finding methods", Appl. Math. Comput., vol. 233, pp. 29-38, 2014.

[10] A. A. Magreńãn, "A new tool to study real dynamics: The convergence plane", Appl. Math. Comput., vol. 248, pp. 29-38, 2014.

[11] E. Martínez, S. Singh, J. L. Hueso, and D. K. Gupta, "Enlarging the convergence domain in local convergence studies for iterative methods in Banach spaces", Appl. Math. Comput., vol. 281, pp. 252-265, 2016.

[12] W. C. Rheinboldt, "An adaptive continuation process for solving systems of nonlinear equations", In: Mathematical models and numerical methods (A.N.Tikhonov et al. eds.) pub.3, pp. 129-142, 1977, Banach Center, Warsaw, Poland.

[13] S. Singh, D. K. Gupta, E. Martínez, and J. L. Hueso, "Semilocal Convergence Analysis of an Iteration of Order Five Using Recurrence Relations in Banach Spaces", Mediterr. J. Math., vol. 13, pp. 4219-4235, 2016. 
[14] S. Singh, E. Martínez, A. Kumar, and D. K. Gupta, "Domain of existence and uniqueness for nonlinear Hammerstein integral equations", Mathematics, vol. 8, no. 3, 2020.

[15] J. F. Traub, Iterative methods for the solution of equations, AMS Chelsea Publishing, 1982.

[16] X. Wang, J. Kou, and C. Gu, "Semi-local convergence of a class of Modified super Halley method in Banach space", J. Optim. Theory. Appl., vol. 153, pp. 779-793, 2012.

[17] Q. Wu, and Y. Zhao, "Newton-Kantorovich type convergence theorem for a family of new deformed Chebyshev method", Appl. Math. Comput., vol. 192, pp. 405-412, 2008.

[18] Y. Zhao, and Q. Wu, "Newton-Kantorovich theorem for a family of modified Halley's method under Hölder continuity conditions in Banach space", Appl. Math. Comput., vol. 202, pp. 243-251, 2008.

[19] A. Emad, M. O. Al-Amr, A. Yıldırım, W. A. AlZoubi, "Revised reduced differential transform method using Adomian's polynomials with convergence analysis", Mathematics in Engineering, Science \& Aerospace (MESA), vol. 11, no. 4, pp. 827-840, 2020. 